\title{
Embarazos en 48 Pacientes
}

\section{Hiperprolactinémicas}

\author{
Dr. Fabio Sánchez Escobar \\ Dr. Jorge Mario Mejía Restrepo
}

Dr. Guillermo Henao Cortés

Dr. Gabriel Devia Manjarrés

\author{
Grupo de Ginecología Endocrina y \\ Reproducción Humana. \\ Departamento de Obstetricia y Ginecología. \\ Centro de Investigaciones Médicas. \\ Facultad de Medicina - Universidad de \\ Antioquia.
}

\section{INTRODUCCION}

Los estados de hiperprolactinemia se asocian frecuentemente a esterilidad como consecuencia de anovulación (1-2), cuerpo lúteo insuficiente $(3-4)$ y posiblemente hostilidad cervical, de acuerdo con la mayor o menor severidad del problema.

La hiperprolactinemia desencadena una perturbación del funcionamiento del hipotálamo y como consecuencia una alteración del mecanismo de retroacción positiva de los estrógenos (5-6), la cual bloquea a la vez la respuesta al clomifeno (7). A nivel hipofisiario puede ejercer un efecto inhibitorio directo sobre la síntesis de gonadotrofinas (8-9-10), indirectamente inhibiendo la $\mathrm{Gn} \mathrm{RH}$ (11) o aumentando la refractariedad ovárica de ellas (12); esto último mediante el freno de la maduración folicular
Hospital San Vicente de Pául.

Medellín - Colombia.

Reimpresos:

Dr. Fabio Sánchez Escobar

Departamento de Obstetricia y Ginecología

Hospital San Vicente de Paúl

Medellín - Colombia - S.A.M.

que perturba la esteroidogénesis tanto en el folículo como en el cuerpo lúteo $(13-14)$.

La hiperprolactinemia también aumenta la concentración de dopamina a nivel del haz tubero infundibular del hipotálamo (15) con lo cual ejerce un efecto inhibitorio sobre la síntesis de gonadotrofinas, posiblemente por efecto sobre la síntesis de $\mathrm{Gn} R \mathrm{RH}$; y en forma directa inhibe la LH hipofisiaria (16).

Todos estos mecanismos se conjugan para dar origen a diversos cuadros clínicos que tiene en común los trastornos menstruales, la anovulación $y$, como resultado, la esterilidad.

En algunos informes se hace énfasis en la diversidad del comportamiento de las prolactinomas en relación con el embarazo, de acuerdo con el tratamiento 
médico (17-18-19-20) o quirúrgico $(21-22)$.

Con el empleo reciente de drogas dopaminérgicas se ha logrado una mayor frecuencia de embarazos en pacientes hiperprolactinémicas, como consecuencia de la regulación neuroendocrina y la normalización de la prolactinemia plasmática $(23-24)$.

Desde hace cuatro años hemos utilizado la bromoergocriptina en forma experimental y sólo desde hace dos años, con su comercialización en nuestro pa ís, se ha utilizado en todas las pacientes con trastornos endocrinos secundarios a hiperprolactinemia.

El presente informe tiene como objetivo presentar nuestra experiencia y los resultados obtenidos en el tratamiento de estas pacientes.

\section{Material y Métodos}

Se analizan los resultados obtenidos en 48 pacientes embarazadas con hiperprolactinemia, de las cuales 27 fueron tratadas en el Grupo de Ginecología Endocrina y Reproducción Humana del, Hospital San Vicente de Paúl, y 21 en el consultorio particular de dos de los autores, localizado en el Centro de Investigaciones Médicas de Antioquia (CIMA) de Medellín.

Se clasifican las pacientes en diferentes entidades, de acuerdo con el cuadro clínico y exámenes complementarios: amenorrea-galactorrea (tumoral, idiopática, post-parto, post-píldoras); síndrome anovulación crónica con estro persistente (o síndrome de ovario poliquístico) y cuerpo lúteo insuficiente. A todas las pacientes se les hizo historia clínica, complementada con exámenes específicos: curva de temperatura basal, citología funcional, análisis de moco cervical, biopsia de endometrio en la época premenstrual o en cualquier época durante el tiempo de amenorrea, previa exclusión de embarazo; determinaciones hormonales por radioinmunoanálisis para prolactina ( $P R L)$. En los casos de atrofia endometrial se solicitan determinaciones de hormona folículo estimulante (FSH) y luteinizante (LH) y determinaciones de 17 cetosteroides en orina de 24 horas. No se cuenta con los medios para determinar pregnandiol en orina o progesterona en plasma.

A las pacientes que tenían concentraciones mayores de $50 \mathrm{ng} / \mathrm{ml}$ de $P$ R L, o alguna sospecha clínica de adenoma de hipófisis, se les practicó tomografía de silla turca anteroposterior y lateral, con cortes cada $2 \mathrm{~mm}$, y campimetría por el método de Goldman.

A todas las pacientes se les practicó la prueba de supresión con la bromoengocriptina con el fin de analizar la respuesta individual y la dósis terapéutica (25).

Se prefirió el tratamiento médico con bromoergocriptina, aunque en algunas pacientes se utilizaron otros esquemas térápeuticos diferentes y como otra alternativa, la cirugía transesfenoidal.

Las muestras de sangre para prolactina fueron recolectadas en tubos de ensayo a la temperatura ambiente $y$ luego coaguladas, se centrifugan y el suero se almacenan a $-20^{\circ} \mathrm{C}$ hasta su estudio por radioinmunoanálisis. Las muestras de cada paciente para la prueba de supresión fueron determinadas con un mismo Kit.

Los niveles de prolactina fueron determinados por medición cuantitativa de precipitación del doble anticuerpo (Prolactin Radioinmunoassay Kit. Sorin Biomedica 13040 Saluggia Vercelli Italia) 


\section{Resultados}

Se estudian un total de 48 pacientes embarazadas con diferentes síndromes de hiperprolactinemia (Tabla 1 - Figura 1). La mayor frecuencia le corresponde al cuerpo lúteo insuficiente $(45.8 \%)$ seguido del síndrome de amenorreagalactorrea $(31.3 \%)$. Sólo una paciente tenía, como única manifestación, la hostilidad cervical y el factor endocrino normal.

Tabla No. 1

PACIENTES HIPERPROLACTI-

NEMICAS EMBARAZADAS

DE ACUERDO CON LA ENTIDAD CLINICA

\begin{tabular}{lcc} 
Diagnóstico & $\begin{array}{c}\text { No. de } \\
\text { pacientes }\end{array}$ & porcentaje \\
\hline Amenorrea-Galactorrea & 15 & 31.3 \\
S. de Ovario Poliquístico & 10 & 20.8 \\
Cuerpo Lúteo Insuficiente & 22 & 45.8 \\
Hostilidad Cervical & 1 & 2.1 \\
\hline Total & 48 & 100.0 \\
\hline & H.U.S.V.P. U de A (1981)
\end{tabular}

En el grupo de pacientes con amenorrea-galactorrea le correspondió la. mayor frecuencia a la causa tumoral 8 , pacientes $(53.3 \%)$, seguido de la causa idiopática, 3 pacientes $(20 \%)$; sólo una paciente tenía el diagnóstico de hipotiroidismo $(6.7 \%)$ (Tabla 2$)$.

48 pacientes tuvieron 50 embarazos; de estos, $47(94 \%)$ fueron por tratamiento médico y sólo $3(6 \%)$ por cirugía transesfenoidal. De las 45 pacientes tratadas médicamente, $34(70.8 \%)$ lo fueron con bromoergocriptina; 5 $(10.4 \%)$ con otros métodos terapéuticos y $6(12.5 \%)$ no necesitaron ningún tratamiento pues se embarazaron duran-
Figura No. 1

\section{PACIENTES HIPERPROLACTI. NEMICAS EMBARAZADAS DE ACUERDO CON LA ENTIDAD CLINICA}

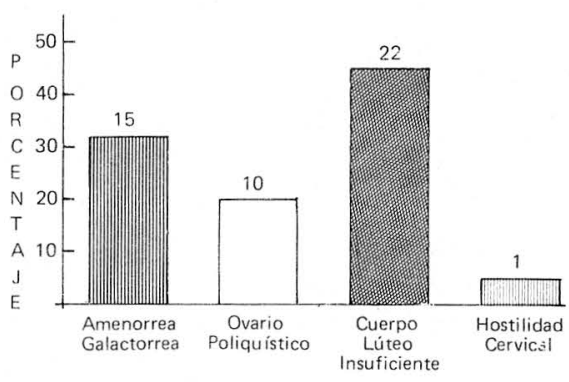

H.U.S.V.P U de A $(1981)$

Tabla No. 2

PACIENTES EMBARAZADAS CON DIAGNOSTICO DE AMENORREA GALACTORREA

Diagnóstico $\begin{gathered}\text { No.de } \\ \text { pacientes }\end{gathered}$ Porcentaje

\begin{tabular}{llr}
\hline Idiopática & 3 & 20.0 \\
Postparto & 2 & 13.3 \\
Postpíldoras & 1 & 6.7 \\
Tumoral & 8 & 53.3 \\
Hipotiroidismo & 1 & 6.7 \\
\hline Total & 15 & 100.0 \\
\hline
\end{tabular}

H.U.S.V.P $-U$ de A (1981)

te el período de estudio (dos postbiopsia de endometrio, dos al suspender la medicación con bromoergocriptina 
Tabla No. 3

\section{TRATAMIENTO EFECTUADO A 48 PACIENTES HIPERPROLACTINEMICAS EMBARAZADAS}

\begin{tabular}{|c|c|c|c|c|c|c|c|c|}
\hline \multirow[b]{2}{*}{ Diagnóstico } & \multirow[b]{2}{*}{$\begin{array}{c}\text { No. de } \\
\text { Pacientes }\end{array}$} & \multicolumn{7}{|c|}{$T E R A P I A$} \\
\hline & & En el estudio & Bromo & L-Dopa & Clomifeno & $\begin{array}{l}\text { L-Dopa } \\
\text { Clomifeno }\end{array}$ & Proloid & Cirugía \\
\hline \multicolumn{9}{|l|}{ Amenorrea Galactorrea } \\
\hline Idiopática & 3 & - & 2 & - & - & 1 & - & - \\
\hline Postparto & 2 & - & 2 & - & - & - & - & - \\
\hline Postpildoras & 1 & - & 1 & - & - & - & - & - \\
\hline Tumoral & 8 & - & - & - & - & - & - & 3 \\
\hline Hipotiroidismo & 1 & - & - & - & - & - & 1 & - \\
\hline S. de Ovario Polĩquístico & 8 & 2 & 6 & 2 & - & - & - & - \\
\hline Cuerpo Lúteo Insuficiente & 22 & 4 & 17 & - & 1 & - & - & - \\
\hline Hostilidad Cervical & 1 & - & 1 & - & - & - & - & - \\
\hline Total & 48 & 6 & 34 & 2 & 1 & 1 & 1 & 3 \\
\hline
\end{tabular}

H. U.S. V.P. - U de A (1981)

y dos durante el estudio). (Tabla 3 figura 2).

Figura No. 2

\section{TRATAMIENTO EFECTUADO A 48 PACIENTES \\ HIPERPROLACTINEMICAS \\ EMBARAZADAS}

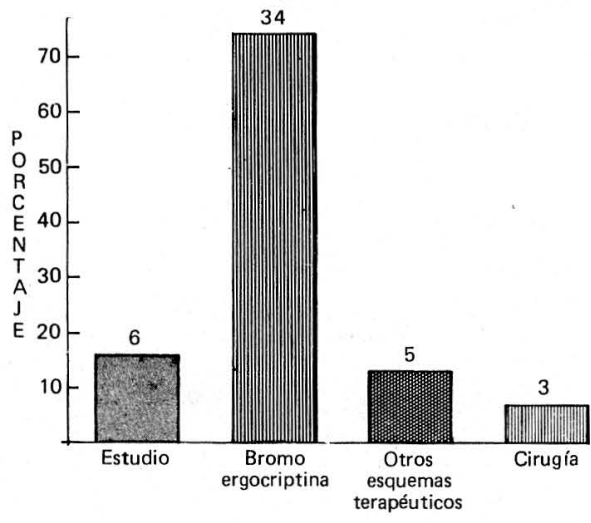

H. U.S. V.P. - U de A (1981)
En las 34 pacientes tratadas con bromoergocriptina, (tabla 4 - figura 3 ) se prescribió la dosis individual luego de la prueba de supresión y en ella se logró un total de 36 embarazos $(72 \%$ del total de embarazos). Ocho se embarazaron durante el ciclo de prueba, sin que se conociera la dosis respuesta, 18 se embarazaron $(50 \%)$ con la dosis de $1,25 \mathrm{mg}$. La mayor respuesta de esta dosis fue el cuerpo lúteo insuficiente, en tanto que los pacientes con amenorrea-galactorrea tumoral necesitaron dosis mayores de bromoergocriptina para embarazarse.

En relación con el tiempo de duración del tratamiento (tabla 5 - figura 4), de las 34 pacientes tratadas con bromoergocriptina hubo 36 embarazos, 8 $(22.22 \%)$ de estos se obtuvieron durante el ciclo de prueba y $20(55.5 \%)$ durante los primeros dos ciclos. Es de resaltar que las pacientes con amenorrea-galactorrea tumoral necesitaron un número mayor de ciclos que las pacientes con otras entidades clínicas para lograr un embarazo. 
Tabla No. 4

RESULTADOS OBTENIDOS DURANTE EL TRATAMIENTO DE 34 PACIENTES CON HIPERPROLACTINEMIA DE ACUERDO A LA DOSIS INDIVIDUAL DE BROMOERGOCRIPTINA

HUS V P - U de A 1979-1981

\begin{tabular}{|c|c|c|c|c|c|c|c|c|}
\hline Diagnóstico & $\begin{array}{l}\text { No. } \\
\text { Ptes. }\end{array}$ & $\begin{array}{l}\text { No. } \\
\text { Embar. }\end{array}$ & $\begin{array}{l}\text { Prueba de } \\
\text { Supres. }\end{array}$ & 1.25 & 250 & 3,75 & 5.0 & 7.5 \\
\hline \multicolumn{9}{|c|}{ Amenorrea-Galactorrea } \\
\hline Idiopática & 2 & 2 & 1 & - & 1 & - & - & - \\
\hline Postparto & 2 & 3 & 3 & - & -- & -- & - & 3 \\
\hline Postpíldoras & 1 & 1 & - & 1 & - & - & - & - \\
\hline Tumoral & 5 & 6 & - & 1 & - & 1 & 3 & 1 \\
\hline S. de ovario Poliquist & t 6 & 6 & 1 & 2 & 1 & 1 & 1 & - \\
\hline \multirow[t]{2}{*}{$\begin{array}{l}\text { Cuerpo lúteo } \\
\text { Insufic. }\end{array}$} & 17 & 17 & 3 & 13 & 1 & - & - & - \\
\hline & 1 & 1 & - & 1 & - & - & - & - \\
\hline Total & 34 & 36 & 8 & 18 & 3 & 2 & 4 & 1 \\
\hline
\end{tabular}

Figura No. 3

RESULTADOS OBTENIDOS DURANTE EL TRATAMIENTO DE 34 PACIENTES CON HIPERPROLACTINEMIA DE ACUERDO CON LA DOSIS INDIVIDUAL

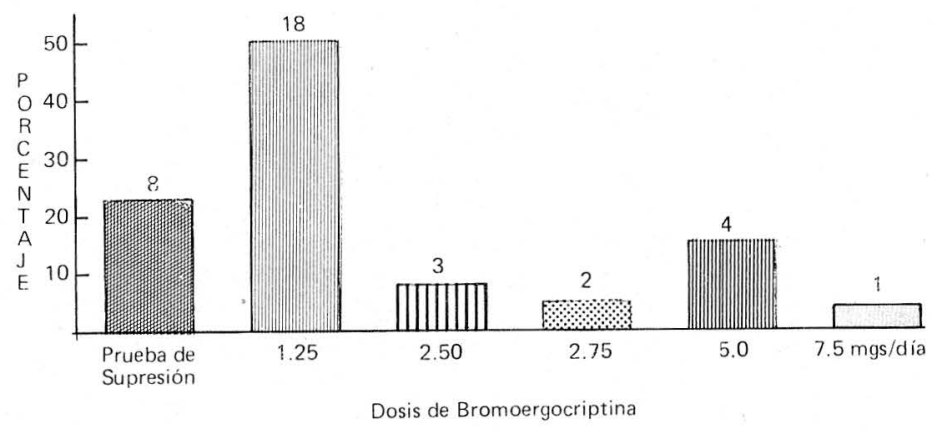

H. U.S. V.P. U de A (1981) 
Tabla No. 5

RESULTADOS OBTENIDOS DURANTE EL TRATAMIENTO DE 34 PACIENTES CON HIPERPROLACTINEMIA DE ACUERDO A LA DURACION DEL TRATAMIENTO CON BROMOERGOCRIPTINA HUS V P $-U$ de A 1976-1979

\begin{tabular}{lcccccccc}
\hline Diagnóstico & No. casos & No. embarazos & $\begin{array}{c}\text { Prueb. de } \\
\text { Supres. }\end{array}$ & 10. & 20. & 3o. & 40. & 50. \\
\hline Amenorrea Galact. & 2 & 2 & 1 & 1 & - & - & - & - \\
Idiopática & 2 & 3 & 3 & - & - & - & - & - \\
Post-parto & 1 & 1 & - & - & 1 & - & - & - \\
Post-p íldoras & 5 & 6 & - & - & - & 2 & 2 & 2 \\
Tumoral & 6 & 6 & 1 & 2 & 3 & - & - & - \\
S. de ovario poliq. & 17 & 17 & 3 & 5 & 7 & 1 & 1 & - \\
Cuerpo lúteo insuf. & 1 & 1 & - & 1 & - & - & - & - \\
Hostilidad cervic. & 34 & 36 & 8 & 9 & 11 & 3 & 3 & 2 \\
\hline \multicolumn{1}{c}{ Total } & & & & & & & & \\
\hline
\end{tabular}

Figura No. 4

RESULTADOS OBTENIDOS DURANTE EL TRATAMIENTO DE 34 PACIENTES CON HIPERPROLACTINEMIA DE ACUERDO CON LA DURACION DEL TRATAMIENTO CON BROMOERGOCRIPTINA

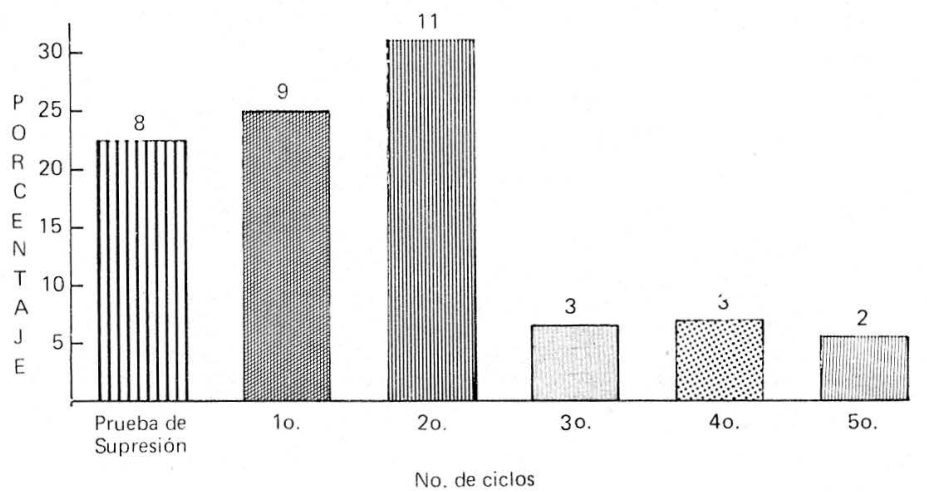

H. U.S. V.P. U de A (1981) 
De los 50 embarazos obtenidos 35 ya concluyeron, así: 3 en aborto $(8.8 \%)$, 4 prematuros $(11.7 \%), 27$ de término $(79.4 \%)$, de uno no se tiene dato; el resto, 15 embarazos en consulta prenatal. No hay actualmente embarazos gemelares (tabla 6).

Tabla No. 6

EVOLUCION DE LOS 50 EMBARAZOS DE PACIENTES HIPERPROLACTINEMICAS

\begin{tabular}{lcc}
\hline & $\begin{array}{c}\text { No. de } \\
\text { Pacientes }\end{array}$ & Porcentaje \\
\hline A término & 27 & 54.0 \\
Prematuros & 4 & 8.0 \\
Abortos & 3 & 6.0 \\
Prenatal & 15 & 30.0 \\
Sin datos & 1 & 2.0 \\
\hline Total & 50 & 100.0 \\
\hline
\end{tabular}

H. U.S. V.P. - U de A (1981)

De los 31 partos, $20(64,6 \%)$ fueron normales y espontáneos y $11(35.4 \%)$ intervenidos, 10 por cesárea y uno con espátulas de Velasco (tabla 7).

24 de los 31 niños son del sexo femenino $(77.4 \%)$ y sólo 7 son del sexo masculino (tabla 8). El peso promedio es de 3.336 .45 gms y la talla $50.4 \mathrm{cms}$.

A un niño se le diagnosticó estenosis pilórica y fue intervenido quirúrgicamente, para un total de $3.2 \%$ de malformaciones congénitas.
Tabla No. 7

TERMINACION DE 31 PARTOS

EN PACIENTES

HIPERPROLACTINEMICAS

\begin{tabular}{|c|c|c|}
\hline & $\begin{array}{c}\text { No. de } \\
\text { pacientes }\end{array}$ & porcentaje \\
\hline $\begin{array}{l}\text { Normal y } \\
\text { espontáneo }\end{array}$ & 20 & 64.6 \\
\hline Espátulas & 1 & 3.2 \\
\hline Cesárea & 10 & 32.2 \\
\hline Total & 31 & 100.0 \\
\hline \multicolumn{3}{|c|}{$\begin{array}{c}\text { Tabla No. } 8 \\
\text { CLASIFICACION DE LOS } 31 \text { NIÑOS } \\
\text { DE PACIENTES } \\
\text { HIPERPROLACTINEMICAS } \\
\text { DE ACUERDO AL SEXO }\end{array}$} \\
\hline Sexo & No. & Porcentaje \\
\hline $\begin{array}{l}\text { Masculino } \\
\text { femenino }\end{array}$ & $\begin{array}{r}7 \\
24\end{array}$ & $\begin{array}{l}22.6 \\
77.4\end{array}$ \\
\hline Total & 31 & 100.0 \\
\hline
\end{tabular}

H. U.S. V.P. - U de A (1981)

\section{Discusión}

De acuerdo con la clasificación de las pacientes hiperprolactinémicas aparece el cuerpo lúteo insuficiente como la entidad más frecuente. En el pasado era ignorada y constituia uno de los motivos diagnósticos de esterilidad de causa desconocida en parejas estériles normales; se diagnostica gracias a la biopsia de 
endometrio (26) y su correlación con la siguiente menstruación (27) y con los cuadros de temperatura basal; su reconocimiento ha permitido un mejor pronóstico a estas parejas (28-29). La severidad del cuadro clínico está relacionada con la mayor concentración de prolactina plasmática; así en 22 pacientes con cuerpo lúteo insuficiente, 'la medida de prolactina es $24.9 \pm 10.4 \mathrm{ng} / \mathrm{ml}$; en el síndrome de ovario poliquístico, en 10 pacientes, $31.5 \pm 13.0 \mathrm{ng} / \mathrm{ml}$ y en los síndromes de amenorrea-galactorrea, concentraciones mayores de $100 \mathrm{ng} / \mathrm{ml}$, encontrándose en estos casos una verdadera atrofia endometrial.

Además en las pacientes con hiperprolactinemia es frecuente encontrar fuera de la patología endocrina (anovulación o cuerpo lúteo insuficiente), una verdadera hostilidad cervical; en 26 pacientes a las cuales se les estudió el moco cervical, $20(77 \%)$ tenían moco de mala calidad, verdaderamente hostil al espermatozoide. Sólo una paciente presentó hostilidad cervical con hiperprolactinemia, asociada a endometrio ovulatorio en fase.

La esterilidad por hiperprolactinemia es actualmente de tratamiento médico, debido al mayor conocimiento de la regulación neuroendocrina y a la utilización de efectivas drogas dopaminérgicas como la bromoergocriptina, que es capaz de normalizar en 2 horas los niveles circulantes de prolactina.

En ausencia de esta droga se utilizan otros esquemas terapéuticos, que actúan a nivel central pero que tienen una muy pobre respuesta, (L Dopa, Clomifeno, $\mathrm{L}$ Dopa + Clomifeno y Clomifeno+ H C G) debido a que la hiperporlactinemia aumenta la refractariedad ovárica (30). La hormona tiroidea únicamente se utilizó en el hipotiroidismo.

Es importante apreciar como el $12 \%$ de los embarazos ocurrieron durante la fase de estudio, posiblemente como consecuencia de cambios endocrinos dest $n$ cadenados por reflejo medular iniciado en genitales, a nivel de cuello o vagina como ocurre en animales (31).

Consideramos a los prolactinomas como entidades de tratamiento médico y sólo en casos especiales en pacientes que presentan intolerancia a la bromoergocriptina, respuesta paradójica, o ausencia de respuesta a ella, se recurre a la cirugía por la vía transesfenoidal, únicamente en los microprolactinomas.

De ocho pacientes con microprolactinoma, a tres se les practicó adenomectomía transesfenoidal. Todas las pacientes embarazadas con hiperprolactinemía fueron controladas estrictamente durante el embarazo: en el primer trimestre, con el fin de diagnosticar y tratar el cuerpo lúteo insuficiente $y$ durante el resto del tiempo controlar y diagnosticar precozmente el crecimiento de un microprolactinoma hipofisiario. Si existen sospechas de cuerpo lúteo insuficiente en una paciente con un embarazo inicial, por temperaturas erráticas, picnosis elevada con mal plegamiento y adosamiento en la citología, y hemorragia leve macro o microscópicamente, se inicia terapia con progesterona de depósito (Proluton) a dosis de 250 a 500 mgs IM, semanal por 15 ó 20 semanas y se controla cada tres semanas, durante las primeras 20 semanas de gestación valorándose citología funcional y curva de temperatura basal. No se puede controlar en nuestro medio el embarazo con determinaciones de pregnandiol en orina o progesterona en plasma. Se proscribe el uso indiscriminado de Proluton.

A las pacientes con prolactinomas, una vez embarazadas, se les suspende el tratamiento con bromoergocriptina y se controlan estrictamente; se tiene especial cuidado con la sintomatología compatible con el crecimiento del tumor tal 
como cefalea de predominio fronto nasal y trastornos visuales, en estos casos, se solicita campimetría cada dos meses. Durante el embarazo no se practica tomografía de silla turca, ni determinaciones de prolactina en suero, por considerar que no son índices pronósticos. Sólo se solicitan durante el postparto y luego cada año en el seguimiento. En sólo tres pacientes fue necesario continuar la terapia con bromoergocriptina durante el embarazo: (una paciente con macroadenoma, una que presentó cefalea fronto-nasal al segundo mes de gestación y una con antecedente de aborto por cuerpo lúteo insuficiente post tratamiento).

La frecuencia de abortos es de $8.8 \%$, porcentaje que no es mayor que el de la población general y coincide con otros informes publicados $y$ muy por debajo al de nuestro hospital $23.7 \%$ en 3.292 embarazos (32). El porcentaje de abortos no fue mayor como se esperaba, posiblemente por el control estricto a que se someten estas pacientes con el fin de diagnosticar precozmente el cuerpo lúteo insuficiente; la presencia de prematurez tuvo una frecuencia más alta con relación a la población general $6.8 \%$, en elnuestro fue de $11.8 \%$ y en nuestro hospital $16 \%$.

En las 31 pacientes que tuvieron su parto, existe una frecuencia elevada de cesáreas $(32.2 \%)$ lo que equivale a que una de cada tres pacientes son intervenidas quirúrgicamente. Porcentaje mu-

\section{Referencias}

1. TZINGOUNIS VA, AKSU MF, TSOUKALES SG (1979). Hyperprolactinemia and polycystic ovarian syndrome. Int $\mathrm{J}$ Fertil 24:276.

2. FALASCHI P, DEL POZO E, ROCCO A, TOSCANO $V$ et al (1980). Prolactin cho más elevado que el $19 \%$ en nuestro hospital (32). El carácter valioso del feto, posiblemente, ha contribuido a que estas pacientes se les considere de alto riesgo $y$ esto ha resultado en que haya un predominio de partos intervenidos por operación cesárea. Las más mínimas complicaciones maternas o fetales inducen al obstetra a tomar esta decisión.

Contrariamente a otras publicaciones, la frecuencia de niños de sexo femenino de $77.4 \%$ es muy alto $(33)$, resultado que or el número tan pequeño (31 niñc.) no tiene validez estadística. Sin embargo puede no ser un hecho aislado, pues la mayoría de estas pacientes presentan un moco hostil, que sería un factor de selección del sexo, El moco, por el tratamiento, adquiere caracteríscas de moco favorable, que posiblemente dependa del tiempo de tratamiento. Como en nuestro estudio la mayoría de las pacientes quedaron en embarazo durante los primeros meses, cuando posiblemente el moco no había adquirido las características de moco óptimo y estaba sometido a la acción directa o indirecta de la prolactina y además la concentración de prolactina puede jugar un papel importante en la capacitación del espermatozoide. Estos factores pudieran explicar este resultado.

No se ha apreciado ningún aumento en la frecuencia de malformaciones congénitas, a pesar de que en algunas pacientes el tratamiento ocasionalmente se ha sostenido hasta las 8 semanas de gestación.

Release in Polycistic Ovary Obstet. Gynecol 55:579.

3. CORENBLUM B, PAIRAUDEAU $N$, SHEWCHUK AB (1976). Prolactin Hypersecretion and Short Luteal Phase defects, Obstet. Gyneco 47:486. 
4. MUHLENSTEDT D, BOHNET HG, HANKER JP, SCHNEIDER HPG (1978). Short Luteal Phase and Prolactin. Int J Fertil 23,213.

5. GLASS MR, SHAW RW, BUTT WR et al (1975). Abnormality of estrogen feedback in amenorrhea - galactorrhea. $\mathrm{Br}$ Med J 3:274.

6. FORSBACH G, SORIA J, CANALES ES, GUZMAN V, CABEZAS A, ZARATE A, (1977). Gonadotropic Responsiveness to $\mathrm{Clomiphene,} \mathrm{LRH}$ and Bromocriptine in Galactorrheic women. Obstet Gynecol 50:139.

7. MC GARRIGLE H H G, SARRIS S, LITTLE V AND COL (1978). Induction of Ovulation With $\mathrm{Clomiphene}$ and $\mathrm{Hu}$ man Chorionic Gonadotroph in in womenwith Hyperprolactinemic amenorrhea. $\mathrm{Br}$ J. Obstet Gynecol 85:692

8. Mc NEILLY AS (1980). Prolactin and the Control of Gonadotrophin Secretion in the Female. J Reprod Fert 58:537.

9. TYSON J E, KHOJANDI-M, HUTH H, et al (1975). The Influence of Prolactin Secretion on Human Laction, Am J. Obstet Gynecol 121:375.

10. SIMPSON CHW, PLUNKETT ER (1979) Pituitary Function Testing in Amenorrhea - Galactorrhea - Hyperprolactinemia. Fertil Steril 32:510.

11. VASQUEZ JM, ELLEGOOD JO, NAZAIN SJ, MAHESH VB (1980). Effect of Hyperprolactinemia on Pituitary Sensibility to Luteinizing Hormone-Releasing Hormone Following Manipulation of sex steroids. Fertil Steril 33:543.

12. ZARATE A, CANALES ES, SORIA J. et al (1972). Ovarian Refractoriness during lactation in women: Effect of gonadotropin stimulation. Am J Obstet Gynecol 112:1130.
13. Mc NATTY KP,. SAWERS RS, Mc. NEILLY AS (1974). A possible role for prolactin in control of steroid secretion by the human Graafian follicle. Nature $250: 653$.

14. ERICKSON G (1978). Función ovárica normal. Clin Obstet Gynecol 1:33

15. BEN - JONATHAN (1980). Cathecolamines and pituitary prolactin release.J. Reprod Fertil 58:501.

16. KAPTEIN EM, KLETZKY OA, SPEN CER CA, NICOLOFF JT (1980). Effects of prolonged dopamine infusion on anterior pituitary function in normal males. J. Clin Endocrinol Metab 51:488.

17. MAGYAR DM, MARSHALL JR (1978). Pituitary tumors and pregnancy. Am J Obstet Gynecol 132:739.

18. LAMBERTS SWJ, KLIJN JGM, DE LANGE SA, et al (1979). The incidence of complications during pregnancy after treatment of hyperprolactinemia with Bromocriptine in patients with radiologically evident pituitary tumor. Fertils Steril 31614.

19. ZARATE A, CANALES ES, ALGER M, FORSBACH G (1979). The effect of pregnancy and lactation on pituitary prolactin - secreting tumours. Acta En docrinol 92:407.

20 SKRABANEK P, McDONALD D, MEAGHER D, DE VALERA E, CARROL C, LANIGAN O, POWELL D (1980). Clinical course and outcome of thirty-five pregnancies in infertile Hyperprolactinemic wornen. Fertil Steril 33:391.

21. KEYE WR, CHANG RJ, MONROE SE, WILSON CB, JAFFE RB (1979). Prolactin - Secreting pituitary adenomas in women. II menstrual function reserves and prolactin production following micro surgical removal. Am J Obstet Gynecol 134:360. 
22. MURRAY FT, OSTERMAN J, SULEWSKI J, et al (1979). Pituitary Function Following surgery for prolactinomas. Obstet Gynecol 54:65.

23. THORNER MO. A Clinical and Pharmacological review, edit Thorner MO, Fluckiger E, Calne DB (1980). Raven Press, New Jork.

24. The dopamine agonist Bromocriptine Theoretical and Clinical Aspects. Edit Hokfelt B, Nillius J (1978). Sandoz Sweden.

25. SANCHEZ F, GARCIA JE, URIBE H, ABAD A: Prueba de supresión con bromoergocriptina como indice diagnóstico y pronóstico (en prensa).

26. NOYES RW, HERTING AT. ROCK J (1950). Dating the endometrial biopsy. Fertil Steril 1:3.

27. JONES GES, MADRIGAL - CASTRO V. (1970). Hormonal Findings in association with abnormal corpus luteum function in the human: The Luteal phase defect. Fertil Steril 21:1.
28. DEL POZO E, WYSS H, TOLIS G ALCAÑIZ J. CAMPANA A, NAFTOLIN $F$ (1979). Prolactin and Deficient luteal function. Obstet. Gynecol 53:282.

29. SAUNDERS DM, HUNTER JCH, Haase HR, Wilson GR (1979). Treatment of luteal phase inadequacy with bromocriptine. Obstet Gynecol 53:287.

30. RADWANSKA E, PHIL M, Mc GARRIGLE HHG, et al (1979). Induction of ovulation in women with Hiperprolactinemic amenorrhea using clomiphene and human Chorionic gonadotropin or Bromocriptine. Fertil Steril 32:187.

31. LINCOLN DW (1969). Response of Hyperthalamic units to stimulation of the vaginal cervix: Specific versus Non Specific effects. J. Endocrinol 43:683.

32. JUBIZ A, CARDONA LE, MANOTAS $R$, CASAS N (1981). Diagnóstico de la situación perinatal en el Hospital Universitario San Vicente de Paúl (Septiembre 80 a mayo 81 ).

33. Sandoz Pharmacenticals. Outcome of pregnancy in mothers taking Parlodel. Status october 31, 1980. 